\title{
A simple fragment-based method for van der Waals corrections over density functional theory
}

\author{
Prasanta Bandyopadhyay, Priya, and Mainak Sadhukhan* \\ Department of Chemistry, Indian Institute of Technology, Kanpur, 208016, India \\ E-mail: mainaks@iitk.ac.in
}

\begin{abstract}
Modelling intermolecular noncovalent interactions between large molecules remains a challenge for electron structure theory community. This is due to the high cost of calculating electron correlation energy. Fragment-based methods usually fare well in reducing the cost of computations in such systems while quantum Drude oscillators turn out to be a good model for van der Waals interactions. In this paper, we have developed a simple yet effective method based on oscillator methods for calculating van der Waals interactions between molecular fragments as a correction to low-cost DFT functional PBE. We have tested our method on S66X8 with significant success.
\end{abstract}

\section{Introduction}

van der Waals (vdW) is a crucial ingredient in understanding the non-covalent interactions between molecular fragments. ${ }^{1-4}$ It originates from instantaneous and correlated quantum fluctuation of electron density electronic systems. As a result, they are ubiquitous and essential to compute with high accuracy. Being long-range part of electron correlation energy, an accurate estimation of the vdW interactions can be obtained from post-Hartree-Fock methods. These methods including coupled-cluster (CC) and configuration interaction (CI) 
calculations provide a systematic and accurate results. However, these methods are computationally too expensive to be used for large molecules particularly for molecular dynamics simulations. On the contrary, density functional theory-based approximations (DFTA) usually fail to describe the vdW interactions properly due to inadequate description of electron correlations. As a result, dispersion corrections are usually employed on top of DFT methods. In this paper, we aim to describe one such correction scheme which is an amalgamation of quantum oscillator based models for quantum fluctuations ${ }^{2,5,6}$ and fragment-based models ( see $^{7}$ for a comprehensive review).

The usual vdW corrections on top of DFT-based methods can be obtained by several strategies. From the perturbation theory, the dispersion energy can be written as a sum of pair-wise terms as, ${ }^{6,8}$

$$
E_{d i s p}^{(2)}=-\sum_{i<j}\left(\frac{C_{6, i j}}{R_{i j}^{6}}+\frac{C_{8, i j}}{R_{i j}^{8}}+\frac{C_{10, i j}}{R_{i j}^{10}}+\cdots\right)
$$

The $\mathrm{i}, \mathrm{j}$ are pair of atoms/systems, $R_{i j}$ is the distance between $\mathrm{i}$ and $\mathrm{j}, C_{n}$ are dispersion coefficients arising from dipole-dipole $(n=6)$, dipole-quadruple $(n=8)$, quadruple-quadruple $(\mathrm{n}=10)$ interactions. In general, the dispersion energy is not pair-wise additive because of the many-body effects. Widely utilized methods for dispersion corrections as Grimme's -D2, ${ }^{9}$ -D $3^{10}$ uses semi-empirical parameters. On the other hand, Tkatchenko-Scheffler method ${ }^{11}$ captures the electronic many-body effects to some extent by defining the electron density based Hirshfeld screening of polarizabilities. Both of these methods has been employed successfully for large molecular systems. ${ }^{12,13}$ The other way of introducing the vdW correction is through quantum Drude oscillator (QDO) model which is a quantum harmonic oscillators with two equal and opposite charges tagged at two ends. As a result, two QDOs interact between themselves through Coulomb interactions. This model has been successfully employed to capture long-range interaction between atoms. ${ }^{6,14-17}$ In particular, dispersion interaction in anionic water-clusters, ${ }^{18,19}$ vibrational spectroscopy in charges water 
clusters, ${ }^{20}$ as a precursor in force-field development, ${ }^{21-23}$ simulating polarizable molecular ionic-liquid $^{24}$ and water-lipid interface ${ }^{25}$ have been investigated using QDO. Many-Body Dispersion (MBD) method ${ }^{5}$ has been another direction where QDO finds its use. In this method, the long-range quantum fluctuations of each atom is modeled by a QDO. The oscillators' polarizabilities have been set to the screened polarizabilities. The dipole-coupled interaction energy between these oscillators therefore provides the interaction energy which is equivalent to random phase approximation (RPA) of correlation energy. ${ }^{26,27}$ Since this model allows for an exact diagonalization of the interaction matrix, it captures many-body effects of all orders. ${ }^{28,29}$

On the other hand, molecular fragmentation techniques have been developed to reduce computational costs for large molecules. Upon fragmentation, a large composite system can be divided into small sub-systems. Thereafter, the results of the high-level calculations on small fragment can be assembled to the desired properties of the large system. ${ }^{7,30}$ Conventional fragment-based methods to calculate the energy of the system can be categorized broadly as overlapping or top-down approach (based inclusion-exclusion principle) and nonoverlapping or bottom-up approach (based on many-body expansion). ${ }^{7}$ MFCC (Molecular Fragmentation with Conjugated Caps), ${ }^{31,32}$ MTA (Molecular Tailoring Approach) ${ }^{33}$, GEBF (Generalized Energy Based Fragmentation) ${ }^{34,35}$ are examples as overlapping fragmentation methods. In these methods, the overcounted fragments are cancelled out to calculate the energy of the derivative subsystems. MFCC utilizes this methodology implicitly. Bottom-up approaches, based upon many-body interactions, first create small non-overlapping fragments to calculate the interactions between them. FMO (Fragment Molecular Orbital), ${ }^{30,36,37}$ EEMB (Electrostatically Embedded Many-Body Method), ${ }^{38,39}$ KEM (Kernel Energy Method) methods ${ }^{40-42}$ follow this methodology. MOBE (Many Overlapping Body expansion) ${ }^{43}$ and GMBE (Generalized Many-Body Expansion) ${ }^{44}$ use both types of top-down and bottomup apprach. In these studies, several fragmentation strategies based on inter-molecular distances, system-size have been employed. Several fragmentation schemes require inter- 
fragment covalent bonds to be cut and be capped that vacancy by atoms (mostly $\mathrm{H}$ ) or a simple group of atoms. This capping makes the charge and multiplicity of the fragments and valence of the adjacent atom intact. Apart from the above mentioned classifications, Richard and Herbert also suggested classification of fragmentation based on intersecting nuclei and disjoint nuclei methods. ${ }^{44}$

In this article, we used an amalgamation of the two above-mentioned methods where after fragmenting the molecule based on simple chemical intuitions, we calculated the longrange vdW interaction energies between them via dipole-coupled quantum Drude oscillators. Such coarse-graining of dispersion interaction helps reduce the computational costs for large molecules. As a result, it is less expensive than that of Dn methods of Grimme as well as traditional oscillator-based methods. We then tested the efficacy of our method on S66x8

dataset. ${ }^{45}$ We found that even this simple procedure provides very good results for most of the systems.

This article is organized as follows. We discussed the theoretical motivations behind using QDO model as well as computational methodologies in section 2. Next, we analysed the fragmentation procedure carefully in section 3.1 followed by the performance of our method for S66X8 database in section 3.2. Then we discussed the performance of other exchange-correlation functionals other than PBE in section 3.3. Finally, we conclude the paper in section 4 .

\section{Theory and Computational Methods}

\subsection{QDO model for dispersion energy}

We start with the definition of electron correlation energy derived from adiabatic connection fluctuation dissipation theorem (ACFDT). ${ }^{5}$ According to this formulation, the electron 
correlation can be written in terms of density response function $\chi$ and the interaction $v .{ }^{26}$

$$
E_{c}=-\frac{1}{2 \pi} \int_{0}^{\infty} d w \int_{0}^{1} d \lambda \quad \operatorname{Tr}\left[\chi_{\lambda} v-\chi_{0} v\right]
$$

Since we are interested in long range correlation energy only, $\mathrm{RPA}^{46,47}$ can be employed to compute the $\mathrm{vdW}$ interactions. In RPA interacting response function $\chi_{\lambda}$ is approximated as:

$$
\chi_{\lambda}^{R P A}=\frac{\chi_{0}}{1-\chi_{0} \lambda v}
$$

In earlier studies ${ }^{26}$ it has been shown that the molecules can be divided into effective atomic fragments. Atomic response functions then can be represented by a set of quantum harmonic oscillators. ${ }^{48}$ Therefore in Eq.(2) can be expressed as

$$
\begin{gathered}
E_{c, R P A}=\frac{1}{2 \pi} \int_{0}^{\infty} d w \operatorname{Tr}[\ln (\mathbf{1}-\mathbf{A T})] \\
=-\frac{1}{2 \pi} \int_{0}^{\infty} d w \sum_{n} \operatorname{Tr}\left[\frac{(\mathbf{A T})^{n}}{n}\right] .
\end{gathered}
$$

where $\mathbf{A}$ is a $3 N \times 3 N$ matrix ( $N$ is number of atoms). For isotropic QHO $\mathbf{A}_{l m}=-\delta_{l m} \alpha_{l}(i w)$ and $\mathbf{T}$ is the dipole-dipole interaction tensor.

We can, however, use a unitary operator $\mathbf{U}$ such that

$$
\mathbf{A}^{\prime}=\mathbf{U A U}^{\dagger} \text { and } \mathbf{T}^{\prime}=\mathbf{U T U}^{\dagger}
$$

Consequently, $\mathbf{A}^{\prime}$ is no longer a diagonal matrix, although

$$
\mathbf{A T}=\mathbf{U}^{\dagger} \mathbf{A}^{\prime} \mathbf{T}^{\prime} \mathbf{U}
$$

leading to

$$
\operatorname{Tr}\left[(\mathbf{A T})^{n}\right]=\operatorname{Tr}\left[\left(\mathbf{A}^{\prime} \mathbf{T}^{\prime}\right)^{n}\right] .
$$


If $\mathbf{A}^{\prime}$ can be constructed in a block-diagonal form, these blocks can be interpreted as fragment polarizabilities and therefore Eq.(4) can be written, in principle, as

$$
E_{c, R P A}=\frac{1}{2 \pi} \int_{0}^{\infty} d w \sum_{n} \operatorname{Tr}\left[\frac{\left(\mathbf{A}^{\prime} \mathbf{T}^{\prime}\right)^{n}}{n}\right]
$$

where $\mathbf{A}^{\prime}$ is the fragment polarizability matrix. However, in this paper we have chosen fragments out of simple chemical intuitions. As a result, our fragments are insensitive to chemical environments. Note that $\mathbf{A}^{\prime}$ defined through Eq.(5) depends on chemical ambiance. More rigorous way of defining polarizabilty fragments based on unitary transformation will be taken up in future. Here we also approximate $\mathbf{T}^{\prime}$ by a simple dipole-dipole interaction matrix between the center-of-masses of two fragments. We modeled the quantum fluctuations of the molecular fragments by anisotropic oscillators. ${ }^{14,26,49}$ The dispersion energies by perturbation theory given by second-order energy. ${ }^{50}$

$$
U_{D i s p}=-\sum_{\mathbf{m}_{A} \neq \mathbf{0}, \mathbf{n}_{B} \neq \mathbf{0}} \frac{\left\langle\mathbf{0}_{\mathbf{A}} \mathbf{0}_{\mathbf{B}}\left|H^{\prime}\right| \mathbf{m}_{\mathbf{A}} \mathbf{n}_{\mathbf{B}}\right\rangle\left\langle\mathbf{m}_{\mathbf{A}} \mathbf{n}_{\mathbf{B}}\left|H^{\prime}\right| \mathbf{0}_{\mathbf{A}} \mathbf{0}_{\mathbf{B}}\right\rangle}{W_{\mathbf{m} \mathbf{0}}^{A}+W_{\mathbf{n} \mathbf{0}}^{B}} .
$$

Here $\mathbf{m}_{\mathbf{i}}=\left(m_{x}, m_{y}, m_{z}\right)$ is an excited state of $i$ oscillator. This expression gives the dispersion energy of electronic fragments A and B. $\mathbf{H}^{\prime}$ is interaction energy operator (see SI) and $W_{\mathbf{m} 0}^{i}=W_{\mathbf{m}}^{i}-W_{\mathbf{0}}^{i}$ is the excitation energy of molecule $i$ with $\mathbf{m}$ quanta.

The detailed derivations and expressions for the dispersion energy using anisotropic oscillators are given in supplementary information (SI).

\subsection{Computational Methodologies}

To benchmark our method, we used S66X8 dataset for its diversity of interaction types. The interaction energy curves from this data-set also help analyzing the effects of fragmentation as well as many-body effects. We have computed all single-point energies using def2-QZVPPD ${ }^{51}$ basis set. The underlying DF interaction energies are calculated as the difference between 
dimer and monomer energies. In this study, several exchange-correlation (XC) functionals including PBE, ${ }^{52}$ PBE0 ${ }^{53}$ B3LYP $^{54,55}$ and BHLYP ${ }^{54,55}$ are used. These XC functionals provide good approximations for short-range interactions. The mid-range and long-range part of correlation energy which constitutes vdW interaction can be computed as,

$$
E_{v d W}=\Delta E_{C C S D(T) / C B S}-\Delta E_{D F}
$$

Here $\Delta E_{C C S D(T) / C B S}$ and $\Delta E_{D F}$ are interaction energies computed by CCSD(T) and approximated density functionals, respectively. Here $\Delta E_{D F}$ contains no dispersion correction. After performing single point energy calculation of the systems at all inter-monomer distances by DF methods, we have spliced the larger monomers into smaller fragments. Detailed fragmentation procedure is mentioned in results and discussion section.

To compute the vdW correction over DF energies, each fragment is replaced by an anisotropic QDO. The dipole-coupled dispersion energies between these oscillators $\left(E_{Q D O}^{i j}\left(r_{i j}\right)\right.$ between $i^{t h}$ and $j^{\text {th }}$ fragments) have been used as the vdW correction energy. The total dispersion energy is obtained by Eq.(11),

$$
E_{v d W}=\sum_{i \in A, j \in B} E_{Q D O}^{i j}\left(r_{i j}\right) f_{d a m p}\left(r_{i j}\right) .
$$

A Fermi-like damping function, ${ }^{56}$

$$
f_{\text {damp }}\left(r_{i j}\right)=\frac{1}{1+\exp \left[-B\left(\frac{r_{i j}}{R_{i j}}-1\right)\right]}
$$

is used to avoid the near-singuarity at very small distance. Parameter $B$ is taken as 6 for optimal performance of the method. $r_{i j}$ is the distance between the centre of mass of the fragments $i$ and $j . R_{i j}$ is the sum of $\mathrm{vdW}$ radii of the fragments $R_{i j}=R_{i}+R_{j}$ obtained $v i a^{57}$

$$
R_{i}(\alpha)=2.54 \alpha^{1 / 7}
$$


Here $\alpha=\operatorname{Tr}\left(\alpha_{i}\right) / 3$, where $\alpha_{i}$ is the polarizability tensor of the $i^{t h}$ fragment. Energies are computed with Turbomole v7.5 58 and static polarizability tensor at CCSD are computed with Psi4. ${ }^{59}$

\section{Results and discussion}

\subsection{Fragmentation of Monomers}

We fragmented the molecular moieties purely based on chemical intuitions (e.g. along the functional groups). Apart from ethene, ethyne and water, all monomers are subjected to fragmentation. As an example, the partitioning of $\mathrm{AcNH}_{2}$ results in two fragments, $-\mathrm{CH}_{3}$ and $-\mathrm{CONH}_{2}$ (Fig.1(a)). The fragments are then capped at the vacant site with $\mathrm{H}$ atoms to keep the charge and spin multiplicity unaltered. The polrizability tensor is computed for these fragments subsequently. Note that a balance between number of fragments and accuracy is imperative from the computational point-of-view. Later in this section, effect of fragmentation and the associated errors are discussed in details.
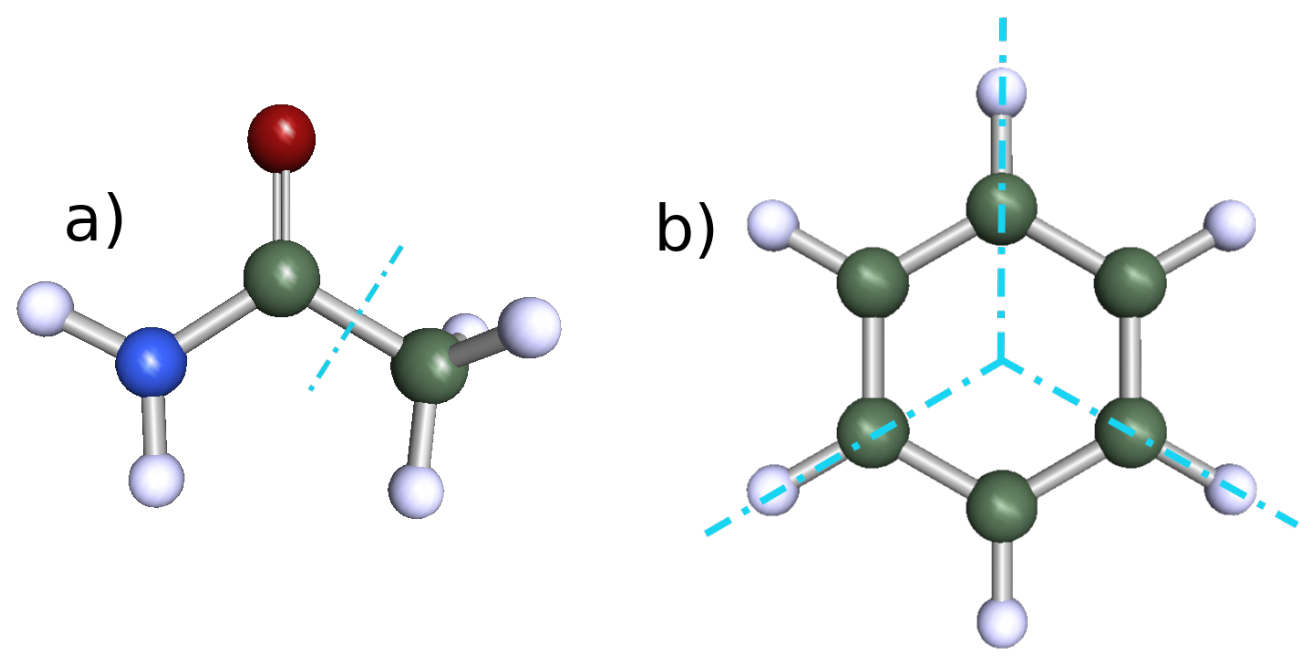

Figure 1: Fragmentation of $\mathrm{AcNH}_{2}$ and Benzene molecule. Green, white, blue and red represents $\mathrm{C}, \mathrm{H}, \mathrm{N}$ and $\mathrm{O}$ atoms, respectively. The cyan dashed lines indicates the line along which fragmentation are performed. 
The monomer fragmentation is done as follows.

- Non-cyclic or non-branched systems are cut and masked with $\mathrm{H}$ atom along the midpoints of bonds (see SI). Fragmenting these molecules in such a manner helps in parametrizing the associated QDOs and therefore provide a scope for transferibility of oscillator parameters across molecules. On the flip side, such fragmentations do not reflect the chemical environment for different molecules. The fragmentation of $\mathrm{AcNH}_{2}$ is shown in Fig.1(a).

- For cyclic molecules, atom-centric fragmentation are performed. As an example, benzene is fragmented along the alternate $\mathrm{C}-\mathrm{H}$ bond (as shown in Fig.1(b) ), thus obtaining three systems of propene. Note two fragments share a bridging atom, thereby making our fragmentation scheme less accurate. However, since the bonds in these systems are electron-rich, the bond-centric fragmentation turns out to be even less accurate. The effects of double-counting atoms will be analyzed and corrected in future works.

\subsection{Performance in $\mathrm{S} 66 \mathrm{x} 8$}

The S66x8 dataset can be categorized into three broad sections, electrostatically bound complexes (named Hydrogen bond by Rezac et $\mathrm{al}^{45}$ ), dispersion-bound and other different interactions. In the following sections, we discuss the role of dispersion in the complexes and how effective our model is to capture this interaction.

\subsubsection{Electrostatically bound complexes}

Electrostatically bound complexes can be characterized by two properties, inter-monomer distance and very low dispersion/electrostatic ratio (hereafter $D / E$ ) as computed from SAPT analysis. In most cases, the $D / E<0.5 .{ }^{45}$ Therefore the qualitative pictures for the interaction energies are essentially obtained from DF methods(see Fig.3). The -D2, -D3 and QDO correction on the DF interaction energies provide more accurate PEC. To analyze further, 
the deviation of interaction energy by PBE-D2 $\left(\Delta E_{P B E-D 2}\right)$ and PBE-QDO $\left(\Delta E_{P B E-Q D O}\right)$ from benchmark coupled-cluster energies are plotted in Fig.2(a)) as obtained via

$$
\begin{gathered}
\Delta E_{P B E-Q D O}=\Delta E_{P B E}+E_{Q D O}-\Delta E_{(C C S D(T) / C B S)} \\
\Delta E_{P B E-D 2}=\Delta E_{P B E-D 2}-\Delta E_{(C C S D(T) / C B S)}
\end{gathered}
$$

Except for the Uracil dimer base-pair interactions, deviation from the CCSD(T)/CBS is well within $1 \mathrm{kcal} / \mathrm{mol}$. Out of 23 electrostatically bound complexes, seven complexes show error $\geq 0.5 \mathrm{kcal} / \mathrm{mol}$. Ten systems show slightly overbinding (blue bars) and for other systems we observe underbinding with respect to CCSD(T). On the contrary, PBE-D2 method (red bars) always shows overbinding. The contribution from dispersion is negligible for majority of these complexes (i.e. < 20\%) (Fig.2)(b). However in Peptide..MeOH, Peptide $\cdots \mathrm{MeNH}_{2}$ and Peptide $\cdots$ Peptide complexes $D / E$ lies within 0.5 and 0.56 mirroring elevated QDO dispersion (> 20\%). Furthermore, for $\mathrm{MeNH}_{2}$-dimer, $\mathrm{MeNH}_{2} \cdots$ Peptide and $\mathrm{MeNH}_{2} \cdots \mathrm{MeOH}$ the dispersion contribution from QDO rise to $\approx 20-30 \%(2 \mathrm{kcal} / \mathrm{mol})$ while the $\mathrm{D} / \mathrm{E}$ ratio lies within 0.71 and 0.79 . Therefore our results are in excellent agreement with the benchmark results.

A close inspection of Fig.2 can reveal the nature and origin of the errors resulted in PBE-QDO method. For most of the complexes, our method predicts under-binding. This may occure due to the absence of dipole-quadrupole and higher order terms. Since 3-body $\mathrm{ATM}^{60,61}$ correction is also important in such systems, inclusion of it may further correct our results. The overall deviation of our method along with pure PBE and PBE-D2 from $\operatorname{CCSD}(\mathrm{T})$ is tabulated in Table-1. QDO correction lowers the error of pure PBE significantly. Also for this subset, the QDO correction fares better than that of PBE-D2 method.

The interaction energy curve of $\mathrm{MeOH}$ dimer, as a representative of these class of complexes (fig.3) shows the comparison of interaction energy between CCSD(T), QDO and D2 corrected PBE. Throughout the distance range the PBE-QDO results show better agreement with $\mathrm{CCSD}(\mathrm{T})$ values compared to PBE-D2. The energy from the QDO interaction 


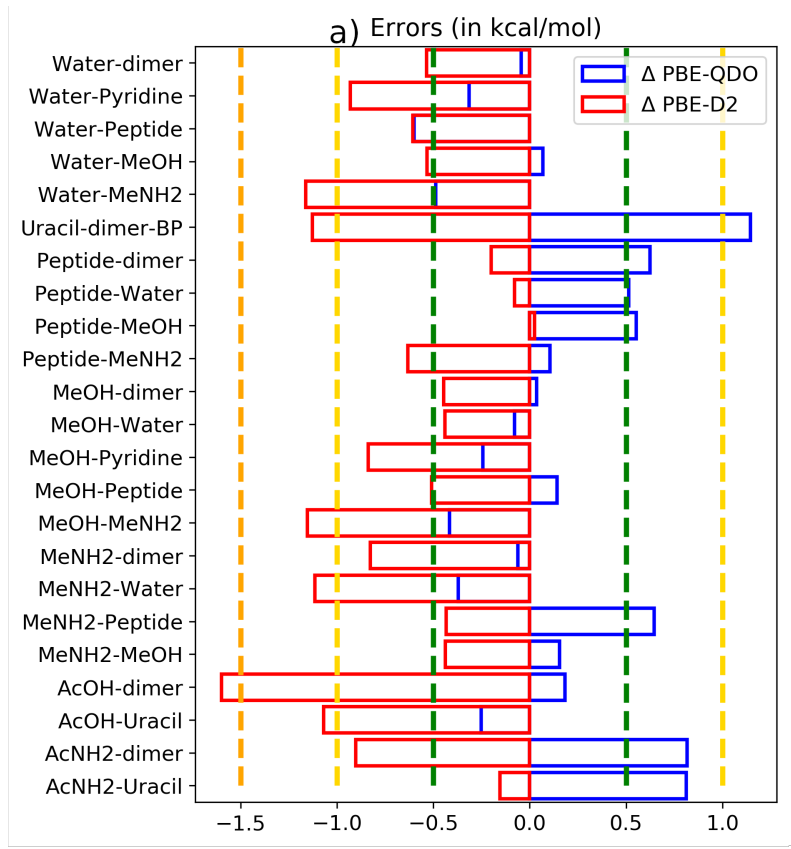

b) \% contribution between QDO and PBE

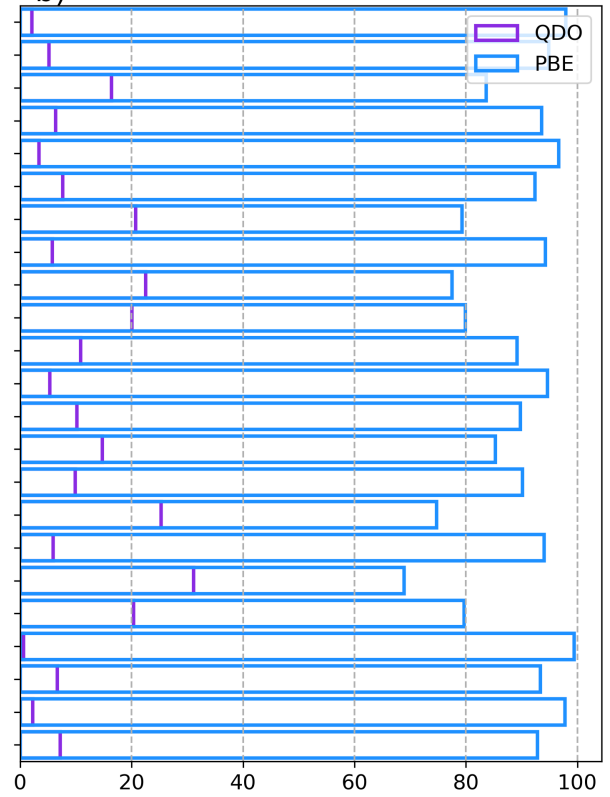

Figure 2: a) $\triangle E_{P B E-Q D O}$ (blue bars) and $\triangle E_{P B E-D 2}$ (red bars) in $\mathrm{kcal} / \mathrm{mol}$ with respect to $\operatorname{CCSD}(\mathrm{T}) / \mathrm{CBS}$ (represented by 0.0 value for abscissa) and b) percentage contributions from $\mathrm{PBE}$ and $\mathrm{QDO}$ dispersion towards PBE+QDO corrected interaction energy at equilibrium distance for electrostatically stabilized complexes.

Table 1: Statistical analysis of error at equilibrium distance with respect to benchmark results for PBE-QDO, PBE and PBE-D2 for electrostatically-bound complexes.

\begin{tabular}{llll}
\hline- & PBE-QDO & PBE & PBE-D2 \\
\hline MAE $^{a}$ & 0.376785 & 0.961688 & 0.684686 \\
MRE $^{b}$ & 0.044944 & 0.124844 & 0.089156 \\
MRAE $^{c}$ & 0.002022 & 0.005149 & 0.003348
\end{tabular}

${ }^{a}$ Mean Absolute Error; ${ }^{b}$ Mean Relative Error; ${ }^{c}$ Mean Relative Absolute Error 
is notable near equilibrium distance $\approx 1 \mathrm{kcal} / \mathrm{mol}$. At the long range, QDO contribution is negligible. PBE-D2 is found to be marginally overestimating the energy with respect to $\operatorname{CCSD}(\mathrm{T}) / \mathrm{CBS}$.

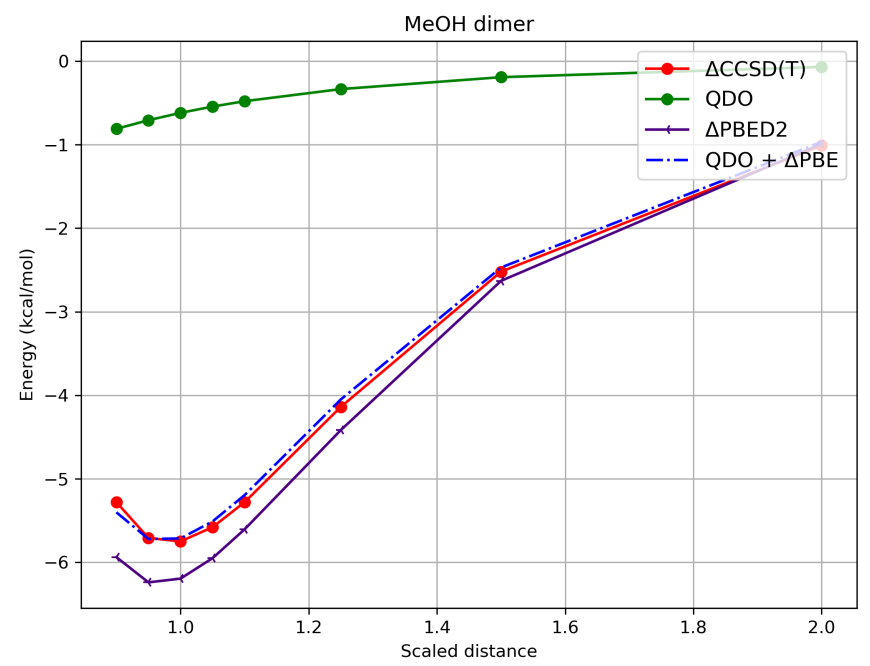

Figure 3: Interaction energy curve (in $\mathrm{kcal} / \mathrm{mol}$ ) for $\mathrm{MeOH}$ dimer plotted against the scaled distance between fragments.

\subsubsection{Dispersion complexes}

Complexes with large $\mathrm{D} / \mathrm{E}$ ratio $(1.3<D / E<5.42)$ are categorized as dispersion complexes. These complexes include stacked dimers. Indeed Fig.5(b) shows relatively large QDO correction compared to DF interaction energies. It is evident that PBE functionals, by themselves do not predict any binding for these complexes. As expected for such cases, QDO correction is the most significant source of binding energies. In fact, for most of the complexes QDO contribution is very prominent.

As a representative of these complexes, we are exhibiting the interaction energy curve of cyclopentane dimer in fig.4. We can clearly see that the QDO contribution is very prominent near equilibrium distance. Moreover, our method consistently agree well with benchmark interaction energies at all distance. It is interesting to note that while D2 correction fares well in long-range, near equilibrium our method is far superior than it. In dispersion bound 


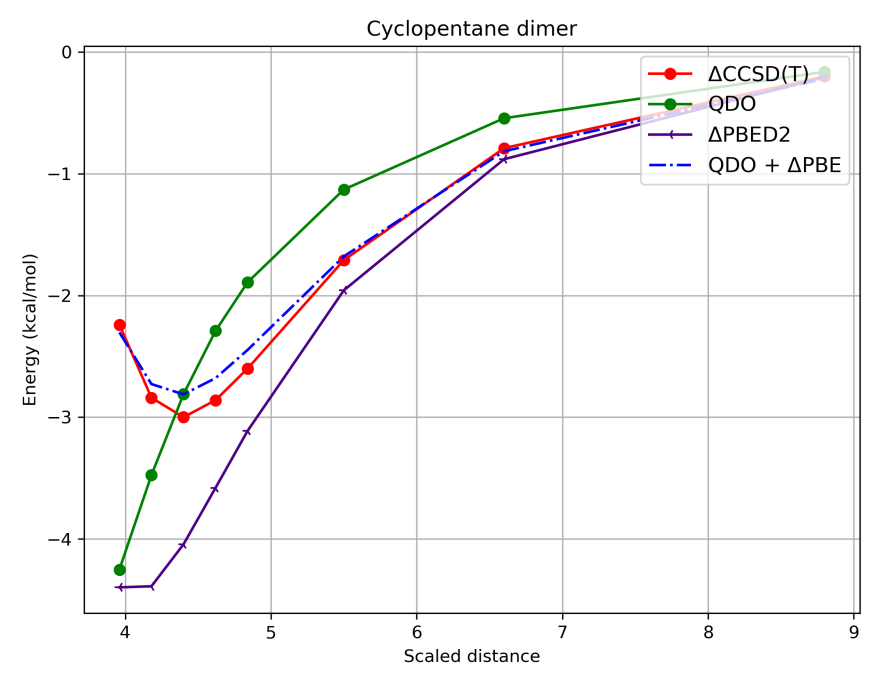

Figure 4: Interaction energy curve (in kcal $/ \mathrm{mol}$ ) for Cyclopentane dimerplotted against the scaled distance between fragments.

complexes, we obtain both under- and overestimated interaction energies which are plotted in Fig.5(a). In most of the pi-pi stacking complexes interaction energy is over-estimated. Apart from these, uracil-cyclopentane and uracil-pentane also show overestimation of interaction energy to some extent. One of the possible causes may include non-optimal choice for the fragmentation method used in the present work. The results were deviated from benchmark numbers most for Benzene-dimer, Benzene-Pyridine and Pyridine-dimer complexes where the monomers are stacked (or parallel-displaced) over each other. To estimate the error associated with this fragmentation procedure, we have calculated the polarizability tensor of all the fragments and monomers at CCSD/def2-TZVPPD. According to Eq.(5), the $\operatorname{Tr}\left(\mathbf{A}^{\prime}\right)=$ $\operatorname{Tr}(\mathbf{A})$. We have been motivated by this to find out the difference between the sum of diagonal elements of $n$ fragment polarizability tensors (i.e. $\alpha_{\mu}$ for $\mu^{\text {th }}$ fragment) and trace of monomer polarizability tensor $\left(\alpha_{\text {monomer }}\right)$ as

$$
\Delta \alpha=\sum_{\mu=1}^{n} \operatorname{Tr}\left(\alpha_{\mu}\right)-\operatorname{Tr}\left(\alpha_{\text {monomer }}\right)
$$


and their ratio

$$
r_{\alpha}=\frac{\sum_{\mu=1}^{n} \operatorname{Tr}\left(\alpha_{\mu}\right)}{\operatorname{Tr}\left(\alpha_{\text {monomer }}\right)}
$$

Clearly, an optimum fragmentation is expected to provide the $r_{\alpha}$ that is close to unity. We can see from the table 2 that the systems with large deviations from the benchmark numbers are also associated with $r_{\alpha}>>1$. Therefore for stacking complexes the error in QDO interaction energies can be attributed to errors in fragmentations.

Table 2: Estimates of errors in fragmentation of monomers. All legends are explained in text (see Eq.(15) and Eq.(16)).

\begin{tabular}{ccccc} 
Monomers & $n^{a}$ & $\operatorname{Tr}\left(\alpha_{\text {monomer }}\right)$ & $\Delta \alpha$ & $r_{\alpha}$ \\
\hline \hline AcNH $_{2}$ & 2 & 117.96 & 15.3 & 1.13 \\
$\mathrm{AcOH}$ & 2 & 102.67 & 14.92 & 1.14 \\
$\mathrm{MeNH}_{2}$ & 2 & 75.54 & 15.37 & 1.20 \\
MeOH & 2 & 62.62 & 15.6 & 1.25 \\
Benzene & 3 & 204.35 & 148.58 & 1.72 \\
Cyclopentane & 3 & 174.39 & 147.45 & 1.84 \\
Pyridine & 3 & 188.87 & 156.64 & 1.83 \\
Uracil & 3 & 211.26 & 131.03 & 1.62 \\
Pentane & 4 & 191.36 & 143.43 & 1.76 \\
Peptide & 4 & 152.88 & 41.12 & 1.27 \\
Neopentane & 4 & 190.52 & 145.03 & 1.76 \\
\hline \multicolumn{5}{c}{ Number of fragments. }
\end{tabular}

For rest of the complexes, small under-estimatation of vdW interaction energy is observed. Notably, the interaction energy for pentane dimer and benzene-ethene complexes are underestimated by $1 \mathrm{kcal} / \mathrm{mol}$. Overall, these large deviations in dispersion interactions can be attributed to the lack of Axilrod-Teller-Muto (ATM) ${ }^{60,61}$ correction and higher order attractive correction terms. Note that most of our corrections has opposite signs than that of PBE-D2 in contrast to electrostatically bound cases discussed before. While it is difficult to pinpoint the reasons for this observation, we can guess that the inclusion of higher order terms may eventually correct for this discrepancy. From statistical viewpoint, both -D2 and QDO dispersion correction reduces the error of PBE (Table -3), while -D2 method is slightly better than QDO corrections. 
Table 3: Statistical analysis of error at equilibrium distance with respect to benchmark results for $\mathrm{PBE}-\mathrm{QDO}, \mathrm{PBE}$ and $\mathrm{PBE}-\mathrm{D} 2$ for dispersion-bound complexes.

\begin{tabular}{llll}
\hline- & PBE-QDO & PBE & PBE-D2 \\
\hline MAE $^{a}$ & 0.601501 & 3.665022 & 0.502804 \\
MRE $^{b}$ & 0.207462 & 1.056156 & 0.158808 \\
MRAE $^{c}$ & 0.008470 & 0.042752 & 0.006559
\end{tabular}

${ }^{a}$ Mean Absolute Error; ${ }^{b}$ Mean Relative Error; ${ }^{c}$ Mean Relative Absolute Error

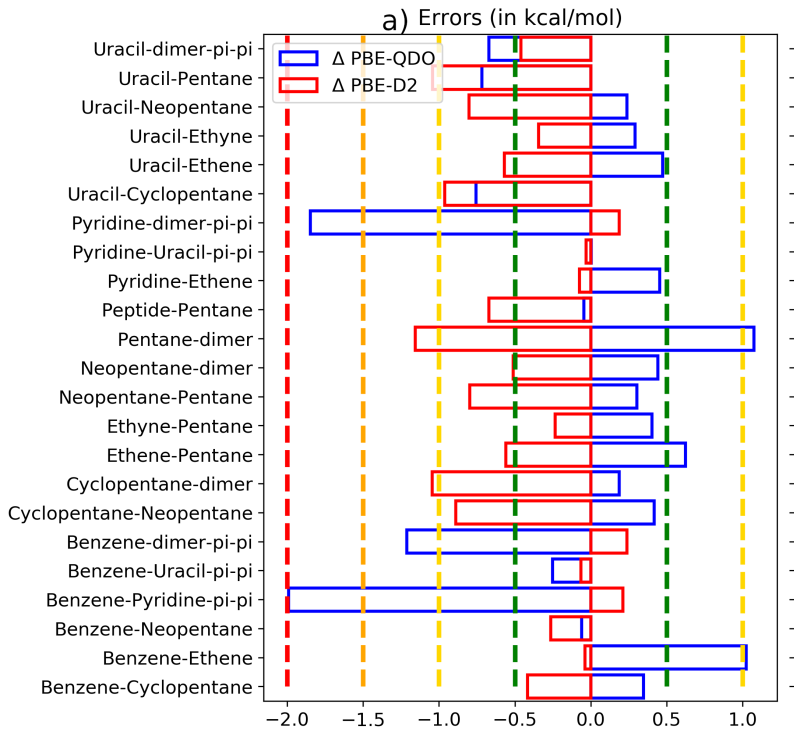

b) \% contribution between QDO and PBE

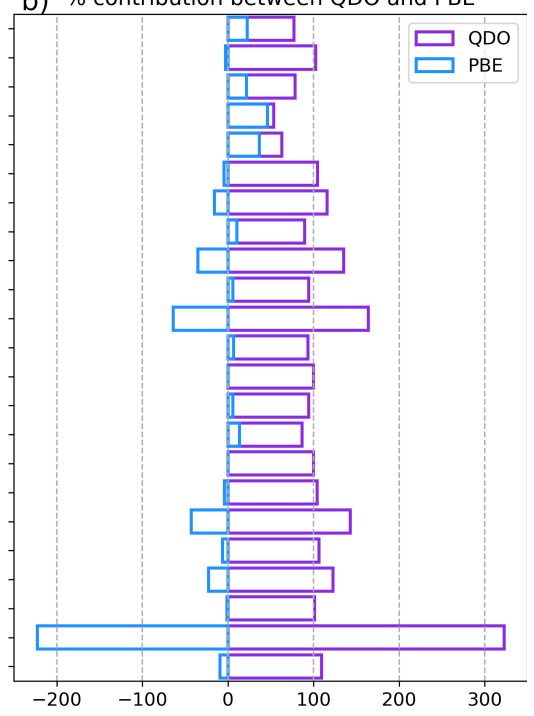

Figure 5: a) $\Delta E_{P B E-Q D O}$ (blue bars) and $\Delta E_{P B E-D 2}$ (red bars) in $\mathrm{kcal} / \mathrm{mol}$ with respect to $\operatorname{CCSD}(\mathrm{T}) / \mathrm{CBS}$ (represented by 0.0 value for abscissa) and b) percentage contributions from $\mathrm{PBE}$ and QDO dispersion towards PBE+QDO corrected interaction energy at equilibrium distance for dispersion stabilized complexes. 


\subsubsection{Other interactions}

Complexes within this category interacts mostly via $\pi$-electrons. Here we show the interaction energy curve of Benzene-water dimer as our representative system(fig.7). As expected, the magnitude of QDO correction here is in between those from previous two sections. While the error due to D2 correction is not much, our correction is signficantly better than D2 here. However, here the D2 approximates the long-range correction much better compared to our method. This may be due to the change of polarizability of fragments at different intermonomer distances which is not considered in our method. In future, therefore, such information should be incorporated in our method. Similar to previously mentioned systems, interaction energy by DF approximations gets better with QDO correction. The deviations of PBE-QDO and PBE-D2 from the CCSD(T) are presented in Fig.6(a). Our results deviate from $\operatorname{CCSD}(\mathrm{T})$ by more than $1 \mathrm{kcal} / \mathrm{mol}$ only for ethyne-AcOH and peptide-ethene. For majority of complexes, PBE-QDO predicts under-binding. This feature has also been observed for dispersion-dominated complexes. As expected, for systems with large dispersion energy component, the QDO correction becomes more significant (Fig.6(b)). We find that for most of these systems dispersion correction constitutes $<40 \%$ of total binding energies. Statistically, (Table-4) we find that both D2 and QDO improves PBE binding energies. However, our method appears to work slightly better than D2.

Table 4: Statistical analysis of error at equilibrium distance with respect to benchmark results for PBE-QDO, PBE and PBE-D2 for mixed complexes.

\begin{tabular}{llll}
\hline- & PBE-QDO & PBE & PBE-D2 \\
\hline MAE $^{a}$ & 0.459231 & 1.76766 & 0.630254 \\
MRE $^{b}$ & 0.134138 & 0.49693 & 0.175168 \\
MRAE $^{c}$ & 0.007765 & 0.026944 & 0.010483 \\
\hline
\end{tabular}

${ }^{a}$ Mean Absolute Error; ${ }^{b}$ Mean Relative Error; ${ }^{c}$ Mean Relative Absolute Error 


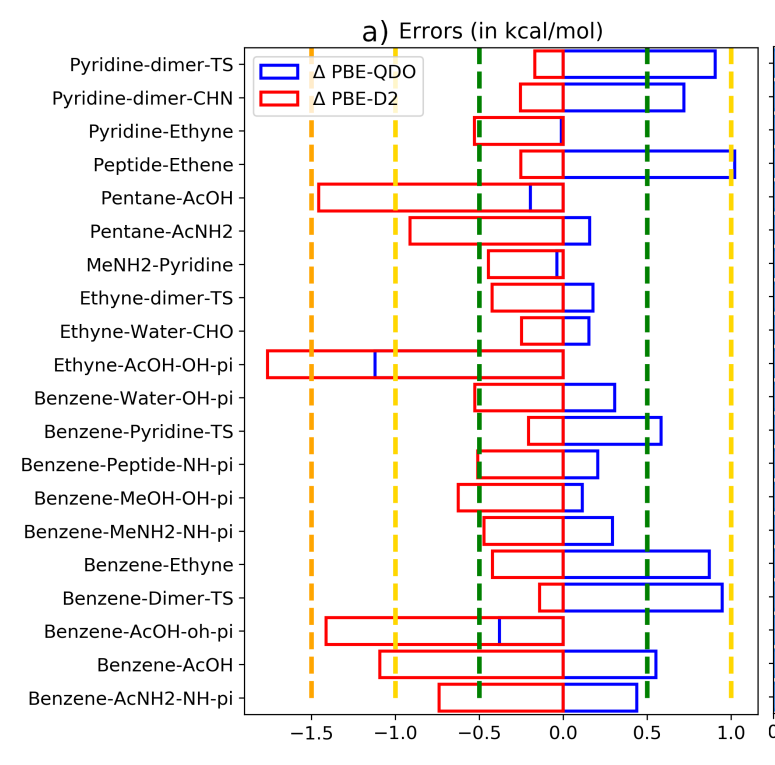

b) \% contribution between QDO and PBE

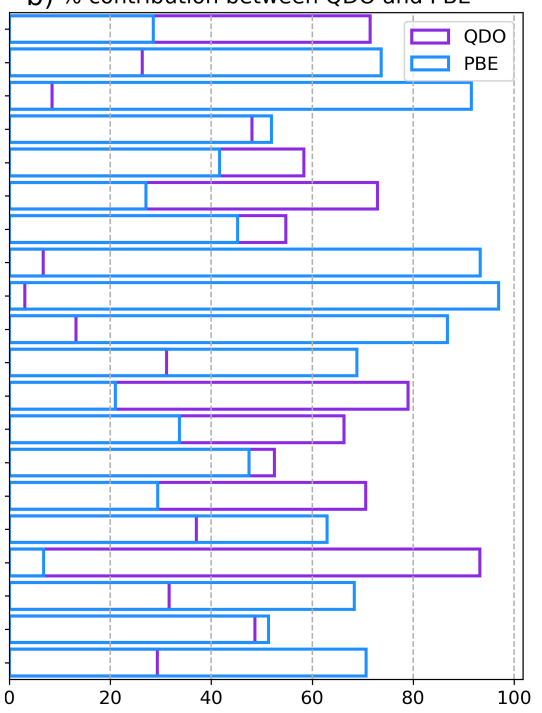

Figure 6: a) $\triangle E_{P B E-Q D O}$ (blue bars) and $\triangle E_{P B E-D 2}$ (red bars) in $\mathrm{kcal} / \mathrm{mol}$ with respect to $\operatorname{CCSD}(\mathrm{T}) / \mathrm{CBS}$ (represented by 0.0 value for abscissa) and b) percentage contributions from $\mathrm{PBE}$ and $\mathrm{QDO}$ dispersion towards $\mathrm{PBE}+\mathrm{QDO}$ corrected interaction energy at equilibrium distance for other type complexes.

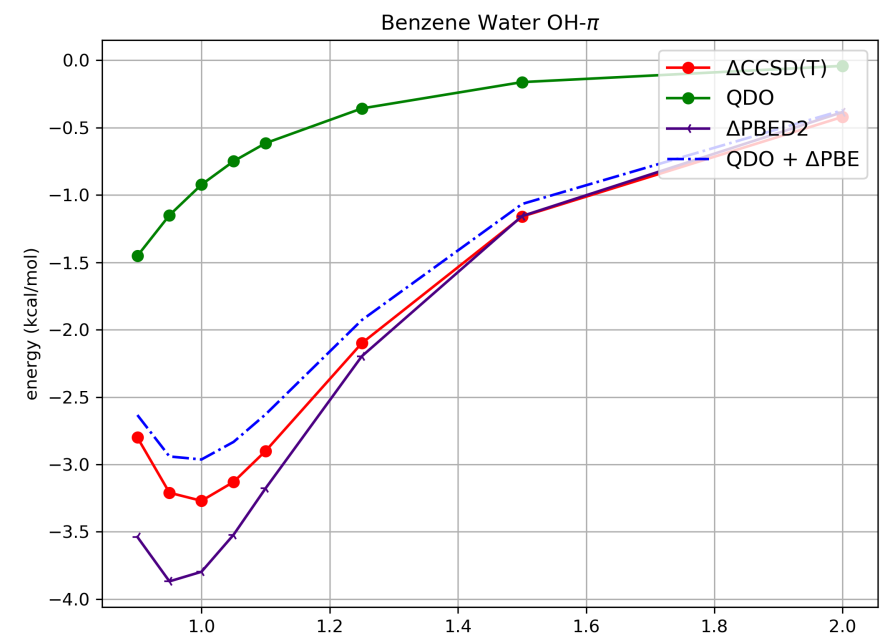

Figure 7: Interaction energy curve (in $\mathrm{kcal} / \mathrm{mol}$ ) for Benzene $\cdots$ Water $\mathrm{OH}-\pi$ complex plotted against the scaled distance between fragments. 


\subsubsection{Overall performance}

The overall error in terms of MAE, MRE and MRAE for all the complexes are tabulated in Table-5. In terms of accuracy, the interaction energy of PBE-QDO is rather accurate. In electrostatic and mixed interaction type complexes, the accuracy is even surpassed than that of the the -D2 correction. Although the accuracy was decreased in dispersion complexes, mostly due to the overestimation of stacking complexes, it is still comparable to -D2. In 66 complexes, only 8 complexes show deviations from $\operatorname{CCSD}(\mathrm{T})$ energy by more than 1 $\mathrm{kcal} / \mathrm{mol}$.

Table 5: Statistical analysis of error at equilibrium distance with respect to benchmark results for PBE-QDO, PBE, PBE-D2, BHLYP-QDO, B3LYP-QDO and PBE0-QDO for all complexes.

\begin{tabular}{lllllll}
\hline- & PBE-QDO & PBE & PBE-D2 & BHLYP-QDO & B3LYP-QDO & PBE0-QDO \\
\hline MAE $^{a}$ & 0.480079 & 2.147993 & 0.604808 & 0.628023 & 1.118247 & 0.516513 \\
MRE $^{b}$ & 0.128608 & 0.562147 & 0.139492 & 0.182652 & 0.323134 & 0.133437 \\
MRAE $^{c}$ & 0.001399 & 0.005983 & 0.001617 & 0.001687 & 0.002810 & 0.001691 \\
\hline
\end{tabular}

${ }^{a}$ Mean Absolute Error; ${ }^{b}$ Mean Relative Error; ${ }^{c}$ Mean Relative Absolute Error

\subsection{Comparison of data with different density functionals}

To analyse the role of different energy components we have applied QDO correction on top of other XC functionals as well. For this we used B3LYP and BHLYP as well as PBE0 functionals. In agreement with Brauer et $\mathrm{al}^{62}$ we find even with QDO correction B3LYP provides very inaccurate binding energies. Furthermore, we tested QDO model with hybrid functional BHLYP which shows better results than B3LYP but worse than PBE. For all of the functionals the inclusion of QDO correction however improves the binding energy. This suggests the exchange energy (since B3LYP and BHLYP both contain LYP correlation) is less important in computing interaction energies around equilibrium geometries. Interaction energy variation with intermonomer distance of all the complexes for these functionals are provided in supporting information. The effect of damping function for different functionals 
remains however an open question which will be taken up in future.

\section{Conclusion}

In summary, we have developed a method to calculate intermolecular dispersion interaction based on a fragmentation technique. We represented the quantum fluctuations of electron densities for these fragments by anisotropic quantum Drude oscillators. It turns out that the method's success heavily depends on the type of fragmentation used. This aspect has been tested with comparing the monomer polarizability with fragment polarizability. We applied our methodology on S66X8 database to understand its efficacy as well as drawbacks. We find that the method works less accurately only for stacked dimers where the electron density is spreadout and therefore highly polarizable. In general, however, this method provides a simple yet effective approximation of dispersion interactions in large molecular systems with accuracy at least at par with popular D2 method. Particularly, we find that except 8 systems, QDO-corrected interaction energy for all other complexes lies within $1 \mathrm{kcal} . / \mathrm{mol}$ of CCSD(T) energy. We also find that our correction due to QDO is maximum for dispersionbound complexes. For such systems, the QDO energy almost provides 100\% binding energy. The mean absolute error also shows that our method works $\approx 20 \%$ better than PBE-D2 methods. Another important aspect of the present work is the use of functional group-based fragmentation scheme. This fragmentation scheme while insensitive to different chemical environments in different systems, is more amenable to application in large systems such as proteins. We also compared the efficacy of different exchange-correlation energies to be used in conjunction to our correction. It turns out that our result, can be used for multiple exchange correlation functionals. However, for B3LYP the correction does not improve the binding energy which is at par with existing literature. The major limitations of our method are twofold: (1) the ad-hoc fragmentation procedure and (2) the lack of screening effects due to local environment. In future, we will investigate ways to include such information 
effectively in our scheme.

\section{Supporting Information Available}

The calculation for the expression of dispersion energy, fragmentation scheme applied for monomers and interaction energy curves for all 66 complexes have been provided.

\section{Acknowledgement}

PB thanks IIT Kanpur for an Institute Post-Doctoral Fellowship. Priya thanks IIT Kanpur for a junior research fellowship. MS thanks SERB grant No. SRG/2019/000369 for computational support and Debashree Manna for numerous stimulating and useful discussions. 


\section{References}

(1) Stöhr, M.; Van Voorhis, T.; Tkatchenko, A. Theory and practice of modeling van der Waals interactions in electronic-structure calculations. Chemical Society Reviews 2019, 48, 4118-4154.

(2) Hermann, J.; DiStasio, R. A.; Tkatchenko, A. First-Principles Models for van der Waals Interactions in Molecules and Materials: Concepts, Theory, and Applications. Chemical Reviews 2017, 117, 4714-4758.

(3) Autumn, K.; Sitti, M.; Liang, Y. A.; Peattie, A. M.; Hansen, W. R.; Sponberg, S.; Kenny, T. W.; Fearing, R.; Israelachvili, J. N.; Full, R. J. Evidence for van der Waals adhesion in gecko setae. Proceedings of the National Academy of Sciences 2002, 99, $12252-12256$.

(4) Rozitis, B.; MacLennan, E.; Emery, J. P. Cohesive forces prevent the rotational breakup of rubble-pile asteroid (29075) 1950 DA. Nature 2014, 512, 174-176.

(5) Tkatchenko, A.; Ambrosetti, A.; DiStasio, R. A. Interatomic methods for the dispersion energy derived from the adiabatic connection fluctuation-dissipation theorem. The Journal of Chemical Physics 2013, 138, 074106.

(6) Jones, A.; Crain, J.; Cipcigan, F.; Sokhan, V.; Modani, M.; Martyna, G. Electronically coarse-grained molecular dynamics using quantum Drude oscillators. Molecular Physics 2013, 111, 3465-3477.

(7) Raghavachari, K.; Saha, A. Accurate Composite and Fragment-Based Quantum Chemical Models for Large Molecules. Chemical Reviews 2015, 115, 5643-5677.

(8) Otero-de-la Roza, A.; LeBlanc, L. M.; Johnson, E. R. What is "many-body" dispersion and should I worry about it? Physical Chemistry Chemical Physics 2020, 22, 82668276. 
(9) Grimme, S. Semiempirical GGA-type density functional constructed with a long-range dispersion correction. Journal of Computational Chemistry 2006, 27, 1787-1799.

(10) Grimme, S.; Antony, J.; Ehrlich, S.; Krieg, H. A consistent and accurate ab initio parametrization of density functional dispersion correction (DFT-D) for the 94 elements H-Pu. The Journal of Chemical Physics 2010, 132, 154104-154104-18.

(11) Tkatchenko, A.; Scheffler, M. Accurate Molecular Van Der Waals Interactions from Ground-State Electron Density and Free-Atom Reference Data. Physical Review Letters 2009, 102, 073005-073005-4.

(12) Cole, D. J.; Vilseck, J. Z.; Tirado-Rives, J.; Payne, M. C.; Jorgensen, W. L. Biomolecular Force Field Parameterization via Atoms-in-Molecule Electron Density Partitioning. Journal of Chemical Theory and Computation 2016, 12, 2312-2323.

(13) Grimme, S.; Antony, J.; Schwabe, T.; Mück-Lichtenfeld, C. Density functional theory with dispersion corrections for supramolecular structures, aggregates, and complexes of (bio)organic molecules. Org. Biomol. Chem. 2007, 5, 741-758.

(14) Voora, V. K.; Ding, J.; Sommerfeld, T.; Jordan, K. D. A self-consistent polarization potential model for describing excess electrons interacting with water clusters. Journal of Physical Chemistry B 2013, 117, 4365-4370.

(15) Sadhukhan, M.; Manby, F. R. Quantum mechanics of Drude oscillators with full Coulomb interaction. Physical Review B 2016, 94, 115106-115106-5.

(16) Odbadrakh, T.; Voora, V.; Jordan, K. Application of electronic structure methods to coupled Drude oscillators. Chemical Physics Letters 2015, 630, 76-79.

(17) Jordan, K. D.; Wang, F. Theory of Dipole-Bound Anions. Annual Review of Physical Chemistry 2003, 54, 367-396. 
(18) Sommerfeld, T.; Jordan, K. D. Quantum Drude Oscillator Model for Describing the Interaction of Excess Electrons with Water Clusters: An Application to (H 2 O) 13 -. The Journal of Physical Chemistry A 2005, 109, 11531-11538.

(19) Sommerfeld, T.; DeFusco, A.; Jordan, K. D. Model Potential Approaches for Describing the Interaction of Excess Electrons with Water Clusters: Incorporation of Long-Range Correlation Effects. The Journal of Physical Chemistry A 2008, 112, 11021-11035.

(20) Tuguldur, T. O. Applications of the Quantum Drude Oscillator Model for Dispersion Interactions and Vibrational Spectroscopy of Charged Water Clusters. 2018; http: //d-scholarship.pitt.edu/34057/.

(21) Chen, T.; Manz, T. A. A collection of forcefield precursors for metal-organic frameworks. RSC Advances 2019, 9, 36492-36507.

(22) Huang, J.; Lopes, P. E. M.; Roux, B.; MacKerell, A. D. Recent Advances in Polarizable Force Fields for Macromolecules: Microsecond Simulations of Proteins Using the Classical Drude Oscillator Model. The Journal of Physical Chemistry Letters 2014, 5, 3144-3150.

(23) Savelyev, A.; MacKerell, A. D. All-atom polarizable force field for DNA based on the classical drude oscillator model. Journal of Computational Chemistry 2014, 35, 12191239.

(24) Schröder, C.; Steinhauser, O. Simulating polarizable molecular ionic liquids with Drude oscillators. The Journal of Chemical Physics 2010, 133, 154511.

(25) Harder, E.; MacKerell, A. D.; Roux, B. Many-Body Polarization Effects and the Membrane Dipole Potential. Journal of the American Chemical Society 2009, 131, 27602761. 
(26) Ambrosetti, A.; Reilly, A. M.; DiStasio, R. A.; Tkatchenko, A. Long-range correlation energy calculated from coupled atomic response functions. The Journal of Chemical Physics 2014, 140, 18A508-18A508-14.

(27) Ambrosetti, A.; Ferri, N.; DiStasio, R. A.; Tkatchenko, A. Wavelike charge density fluctuations and van der Waals interactions at the nanoscale. Science 2016, 351, 11711176.

(28) Stöhr, M.; Tkatchenko, A. Quantum mechanics of proteins in explicit water: The role of plasmon-like solute-solvent interactions. Science Advances 2019, 5, eaax0024.

(29) Claudot, J.; Kim, W. J.; Dixit, A.; Kim, H.; Gould, T.; Rocca, D.; Lebègue, S. Benchmarking several van der Waals dispersion approaches for the description of intermolecular interactions. The Journal of Chemical Physics 2018, 148, 064112.

(30) Gordon, M. S.; Fedorov, D. G.; Pruitt, S. R.; Slipchenko, L. V. Fragmentation Methods: A Route to Accurate Calculations on Large Systems. Chemical Reviews 2012, 112, $632-672$.

(31) Zhang, D. W.; Zhang, J. Z. H. Molecular fractionation with conjugate caps for full quantum mechanical calculation of protein-molecule interaction energy. The Journal of Chemical Physics 2003, 119, 3599-3605.

(32) He, X.; Zhu, T.; Wang, X.; Liu, J.; Zhang, J. Z. H. Fragment Quantum Mechanical Calculation of Proteins and Its Applications. Accounts of Chemical Research 2014, 47, $2748-2757$.

(33) Gadre, S. R.; Shirsat, R. N.; Limaye, A. C. Molecular Tailoring Approach for Simulation of Electrostatic Properties. The Journal of Physical Chemistry 1994, 98, 9165-9169.

(34) Li, W.; Li, S.; Jiang, Y. Generalized Energy-Based Fragmentation Approach for Com- 
puting the Ground-State Energies and Properties of Large Molecules. The Journal of Physical Chemistry A 2007, 111, 2193-2199.

(35) Li, S.; Li, W.; Ma, J. Generalized Energy-Based Fragmentation Approach and Its Applications to Macromolecules and Molecular Aggregates. Accounts of Chemical Research 2014, 47, 2712-2720.

(36) Kitaura, K.; Ikeo, E.; Asada, T.; Nakano, T.; Uebayasi, M. Fragment molecular orbital method: an approximate computational method for large molecules. Chemical Physics Letters 1999, 313, 701-706.

(37) Pruitt, S. R.; Bertoni, C.; Brorsen, K. R.; Gordon, M. S. Efficient and Accurate Fragmentation Methods. Accounts of Chemical Research 2014, 47, 2786-2794.

(38) Dahlke, E. E.; Truhlar, D. G. Electrostatically Embedded Many-Body Correlation Energy, with Applications to the Calculation of Accurate Second-Order MøllerPlesset Perturbation Theory Energies for Large Water Clusters. Journal of Chemical Theory and Computation 2007, 3, 1342-1348.

(39) Wang, B.; Yang, K. R.; Xu, X.; Isegawa, M.; Leverentz, H. R.; Truhlar, D. G. Quantum Mechanical Fragment Methods Based on Partitioning Atoms or Partitioning Coordinates. Accounts of Chemical Research 2014, 47, 2731-2738.

(40) Huang, L.; Massa, L.; Karle, J. Kernel energy method illustrated with peptides. International Journal of Quantum Chemistry 2005, 103, 808-817.

(41) Huang, L.; Krupkin, M.; Bashan, A.; Yonath, A.; Massa, L. Protoribosome by quantum kernel energy method. Proceedings of the National Academy of Sciences 2013, 110, $14900-14905$.

(42) Weiss, S. N.; Huang, L.; Massa, L. A generalized higher order kernel energy approximation method. Journal of Computational Chemistry 2010, 31, 2889-2899. 
(43) Mayhall, N. J.; Raghavachari, K. Many-Overlapping-Body (MOB) Expansion: A Generalized Many Body Expansion for Nondisjoint Monomers in Molecular Fragmentation Calculations of Covalent Molecules. Journal of Chemical Theory and Computation 2012, 8, 2669-2675.

(44) Richard, R. M.; Herbert, J. M. A generalized many-body expansion and a unified view of fragment-based methods in electronic structure theory. The Journal of Chemical Physics 2012, 137, 064113-064113-17.

(45) Řezáč, J.; Riley, K. E.; Hobza, P. S66: A Well-balanced Database of Benchmark Interaction Energies Relevant to Biomolecular Structures. Journal of Chemical Theory and Computation 2011, 7, 2427-2438.

(46) Ren, X.; Rinke, P.; Joas, C.; Scheffler, M. Random-phase approximation and its applications in computational chemistry and materials science. Journal of Materials Science 2012, $47,7447-7471$.

(47) Eshuis, H.; Bates, J. E.; Furche, F. Electron correlation methods based on the random phase approximation. Theoretical Chemistry Accounts 2012, 131, 1084.

(48) Ambrosetti, A.; Reilly, A. M.; DiStasio, R. A.; Tkatchenko, A. Long-range correlation energy calculated from coupled atomic response functions. The Journal of Chemical Physics 2014, 140, 18A508-18A508-14.

(49) Jones, A.; Cipcigan, F.; Sokhan, V. P.; Crain, J.; Martyna, G. J. Electronically CoarseGrained Model for Water. Physical Review Letters 2013, 110, 227801-227801-5.

(50) Stone, A. J. The Theory of Intermolecular Forces, 2nd ed.; Oxford University Press.

(51) Weigend, F.; Ahlrichs, R. Balanced basis sets of split valence, triple zeta valence and quadruple zeta valence quality for $\mathrm{H}$ to Rn: Design and assessment of accuracy. Physical Chemistry Chemical Physics 2005, 7, 3297-3305. 
(52) Perdew, J. P.; Ernzerhof, M.; Burke, K. Rationale for mixing exact exchange with density functional approximations. The Journal of Chemical Physics 1996, 105, 99829985.

(53) Adamo, C.; Barone, V. Toward reliable density functional methods without adjustable parameters: The PBE0 model. The Journal of Chemical Physics 1999, 110, 6158-6170.

(54) Becke, A. D. Density-functional exchange-energy approximation with correct asymptotic behavior. Physical Review A 1988, 38, 3098-3100.

(55) Lee, C.; Yang, W.; Parr, R. G. Development of the Colle-Salvetti correlation-energy formula into a functional of the electron density. Physical Review B 1988, 37, 785-789.

(56) Wu, Q.; Yang, W. Empirical correction to density functional theory for van der Waals interactions. The Journal of Chemical Physics 2002, 116, 515-524.

(57) Fedorov, D. V.; Sadhukhan, M.; Stöhr, M.; Tkatchenko, A. Quantum-Mechanical Relation between Atomic Dipole Polarizability and the van der Waals Radius. Physical Review Letters 2018, 121, 183401-183401-6.

(58) Balasubramani, S. G.; Chen, G. P.; Coriani, S.; Diedenhofen, M.; Frank, M. S.; Franzke, Y. J.; Furche, F.; Grotjahn, R.; Harding, M. E.; Hättig, C.; Hellweg, A.; Helmich-Paris, B.; Holzer, C.; Huniar, U.; Kaupp, M.; Marefat Khah, A.; Karbalaei Khani, S.; Müller, T.; Mack, F.; Nguyen, B. D.; Parker, S. M.; Perlt, E.; Rappoport, D.; Reiter, K.; Roy, S.; Rückert, M.; Schmitz, G.; Sierka, M.; Tapavicza, E.; Tew, D. P.; van Wüllen, C.; Voora, V. K.; Weigend, F.; Wodyński, A.; Yu, J. M. TURBOMOLE: Modular program suite for ab initio quantum-chemical and condensed-matter simulations. The Journal of Chemical Physics 2020, 152, 184107-184107-36.

(59) Smith, D. G. A.; Burns, L. A.; Simmonett, A. C.; Parrish, R. M.; Schieber, M. C.; Galvelis, R.; Kraus, P.; Kruse, H.; Di Remigio, R.; Alenaizan, A.; James, A. M.; 
Lehtola, S.; Misiewicz, J. P.; Scheurer, M.; Shaw, R. A.; Schriber, J. B.; Xie, Y.; Glick, Z. L.; Sirianni, D. A.; O’Brien, J. S.; Waldrop, J. M.; Kumar, A.; Hohenstein, E. G.; Pritchard, B. P.; Brooks, B. R.; Schaefer, H. F.; Sokolov, A. Y.; Patkowski, K.; DePrince, A. E.; Bozkaya, U.; King, R. A.; Evangelista, F. A.; Turney, J. M.; Crawford, T. D.; Sherrill, C. D. PSI4 1.4: Open-source software for highthroughput quantum chemistry. The Journal of Chemical Physics 2020, 152, 184108$184108-21$.

(60) Axilrod, B. M.; Teller, E. Interaction of the van der Waals Type Between Three Atoms. The Journal of Chemical Physics 1943, 11, 299-300.

(61) Muto, Y. Force between nonpolar molecules. J. Phys. Math. Soc. Japan 1943, 17, 629-631.

(62) Brauer, B.; Kesharwani, M. K.; Kozuch, S.; Martin, J. M. L. The S66x8 benchmark for noncovalent interactions revisited: explicitly correlated ab initio methods and density functional theory. Physical Chemistry Chemical Physics 2016, 18, 20905-20925. 


\section{TOC Graphic}

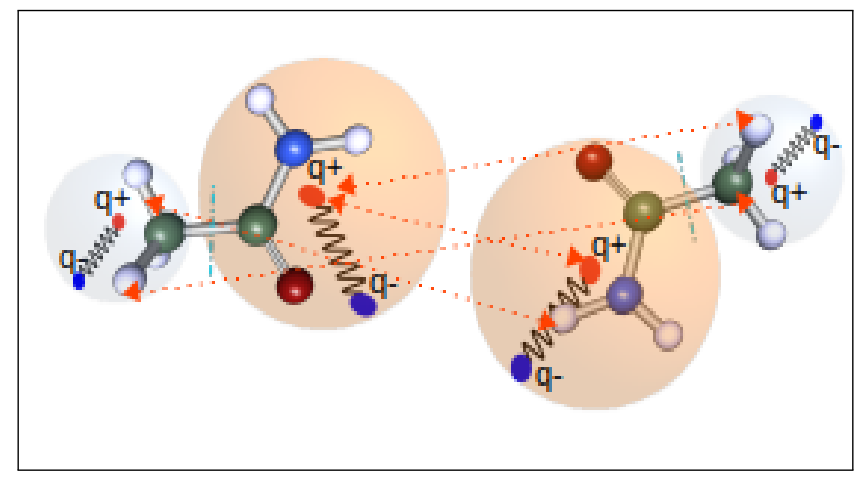

